\title{
Design for Passivity in the Z-Domain for LCL Grid-Connected Converters
}

\author{
Francisco D. Freijedo, Drazen Dujic \\ Ecole Polytechnique Federale de Lausanne - EPFL \\ Power Electronics Laboratory - PEL \\ Email:francisco.freijedo@epfl.ch,drazen.dujic@epfl.ch
}

\author{
Juan Alberto Marrero-Sosa \\ ABB Switzerland Ltd. \\ Traction \\ Email:juan-alberto.marrero-sosa@ch.abb.com
}

\begin{abstract}
This paper develops a design methodology aimed to shape passive the admittance of the LCL grid-connected voltage source converters (VSCs). A novel aspect of this work is the assessment of the range of frequencies for control design: due to discrete and PWM operation, the effectiveness of the control action is more and more reduced as frequency increases; in practice, system delays and non-linear effects tend to impair the passivity properties and also its experimental validation. However, as shown in this paper, those effects can be minimized by including the LCL filter as a part of an outer VSC admittance: this assumption is supported by the fact that high frequency disturbances (generated in the point of connection) are absorbed by the LCL capacitor branch, and hence, are not able to create a positive feedback in the VSC (i.e., the active component). By taking advantage of this remark, the inner VSC admittance can be shaped by a reduced order filter in the $Z$-domain, which mainly depends on the proportional and active damping (controller) gains. The design hypotheses and the control design methodology are verified by PLECS switching-mode simulations.

Index Terms-Ac/dc power conversion, active damping, current control, harmonics, pulse width modulation converters, stability, wind power generation.
\end{abstract}

\section{INTRODUCTION}

Stability of power converters connected to highly variable grid conditions is a challenging issue. This is the case of traction applications, in which the electric circuit seen by the locomotive inverter (i.e., the plant) is continuously changing during operation [1]. Focusing on renewable energy applications, high uncertainty in the plant model is found in large wind farms due to elements such as long cables, capacitor banks and transformers [2], [3]. This work focuses on the dynamics associated to current controlled LCL grid-connected voltage source converters (VSCs), which represent many of the harmonic issues in wind power plants [2].

From the classical closed-loop control perspective, the difficulties to assess the plant (i.e., the electric circuit) makes the design a complex task. Design for passivity successfully deals with a limited knowledge of plant (i.e., the electric circuit in this problem), since the control objectives are set as a function of well known parameters. A design for passivity methodology for grid-connected VSC has been developed in [4], [5]; it can be summarized as follows: 1) the closed loop control is expressed in terms of the impedance stability criterion [6];2) the converter admittance is derived as function of converter parameters (controller and primary inductance); 3) the grid impedance is not known, but it is assumed formed by passive components; 4) from previous remarks, absolute stability is assured if the converter admittance is passive: i.e., its real part is not negative; 5) systematic procedures to set control filters and parameters is provided with the goal of shaping the converter admittance to be passive.

Some features of those previous works are: 1) the design has been developed in the S-domain; 2) the range of frequencies for analysis covers components from the closed loop bandwidth up to the Nyquist frequency, which (by definition) is $\omega_{s} / 2$, with $\omega_{s}$ being the controller sampling frequency [4], [5]. However, as analyzed in this paper, this methodology poses important practical limitations, such as 1) when considering the discrete-time implementation obtained from S-domain filters, significant inaccuracies arise at the high frequency range, which clearly compromise $\mathrm{S}$-domain based designs [7][9]; 2) discrete-time implementation of time-derivative filters, which is also needed in the active damping path, is not straightforward task [10], [11]; 3) non-linear effects due to zero order hold $(\mathrm{ZOH})$ and PWM operation effectively limit the control action [12], which is not considered by a linear analysis; 4) assuming that most of the practical grid-connected VSC include a LCL filter, the high-frequency filtering of the capacitor branch can be taken into account to simplify the design problem without compromising the stability properties.

Based on these observations, this paper proposes to limit the design region into the range of frequencies at which the control active is really effective. As shown in Section III, $\omega_{s} / 4$ is a reasonable limit for the controller design (i.e., the capacitor branch effectively isolates high frequency components to draw the VSC). Subsequently, Sections IV (system modeling) and $\mathrm{V}$ (control design) show that a passive design is achieved with a simple controller by taking consideration of the implementation: the design is based on a Z-domain approach with consideration of sampling and delay effects.

Simulation results are employed to verify the approach. The discrete control system is implemented in Matlab Simulink (discrete time blocks with $\mathrm{ZOH}$ acquisition) with the power circuit switching model implemented with the PLECS blockset. The tests approach the passivity verification procedure set in the EN-50388 standard, available for traction applications [13]: an ac sweep is programmed in the voltage waveform at the point of common coupling and then the admittance frequency characteristic is obtained from the current measurements. The results confirm the proposed approach. 


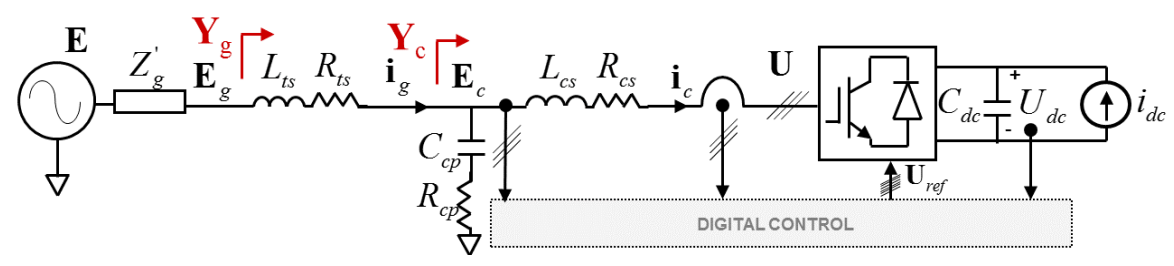

(a)

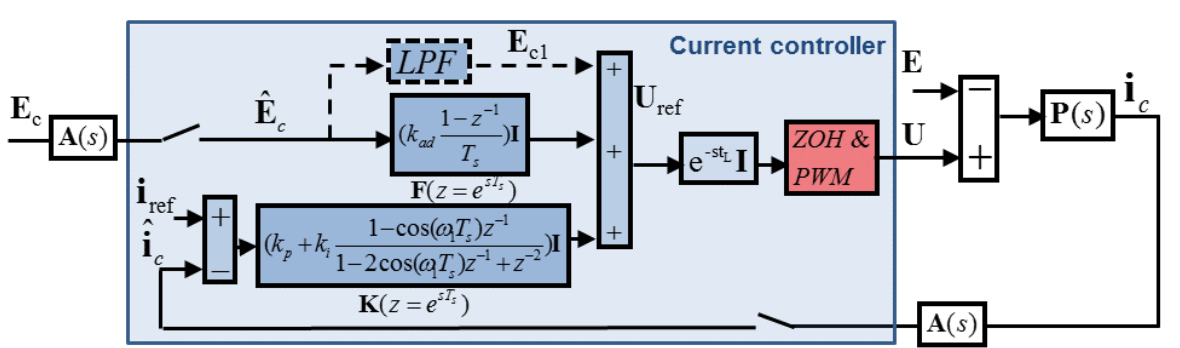

(b)

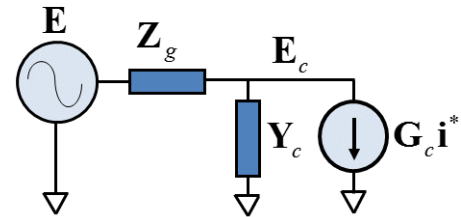

(c)

Fig. 1. System description. (a) LCL grid-connected VSC. (b) Current controller loop. (c) Impedance Stability formulation.

\section{SYSTEM DESCRIPTION AND BACKGROUND}

\section{A. LCL Grid-connected VSC}

Fig. 1(a) represents a LCL grid-connected VSC working in current control mode. The voltages $E, E_{c}$ and $U$ represent the stiff grid, point of connection and VSC voltages, respectively. The LCL output filter is formed by the converter side (primary) inductive filter, the capacitance and transformer leakage inductance. The converter side inductive filter is defined by series inductance $L_{c s}$ and resistance $R_{c s}$. The capacitance is given by a parallel capacitance $C_{c p}$ in series with a small equivalent series resistor $R_{c p}$. The transformer model is given by a leakage inductance $L_{t s}$ and an equivalent winding resistor $R_{t s}$ in series. The grid impedance is represented by $Z_{g}^{\prime}$, which depends on power system circuit and grid conditions [3].

In wind turbine applications, the hardware design is imposed by the transformer leakage inductance, which sets $L_{t s}$ [3], [14]. Typical values for the secondary inductance are then in the range $[0.06,0.1]$ p.u. (wind turbine rated power is used for base calculations) [14]. Following LCL design basic guidelines, the secondary inductance also constraints the selection of the converter filter: a primary inductance equal to the transformer inductance is a reasonable design to optimize the switching harmonics filtering [11], [14]. Using $L_{t s}$ as a constraint, in practice, the main degree of freedom of the LCL filter is the choice of the capacitance $C_{c p}$. The rated LCL resonance frequency (angular) is

$$
\omega_{\text {res }}=\sqrt{\frac{L_{c s}+L_{t s}}{L_{c s} L_{t s} C_{c p}}} .
$$

The selection of $\omega_{\text {res }}$ involves a trade-off between control interactions and filtering [11]. For active damping, typical values at which the capacitor voltage feedback is more effective are in the range $\left[0.1 \omega_{s}, 0.2 \omega_{s}\right][11]$.

\section{B. Current Controller}

Fig. 1(b) depicts the current loop, which corresponds to a hybrid controller [7]. $\mathbf{K}(z)$ represents the main controller, which in this work is a PR implemented in $\alpha \beta$-frame

$$
\mathbf{K}(z)=\left(k_{p}+k_{i} \frac{1-\cos \left(\omega_{1} T_{s}\right) z^{-1}}{1-2 \cos \left(\omega_{1} T_{s}\right) z^{-1}+z^{-2}}\right) \mathbf{I}
$$

with $k_{p}$ and $k_{i}$ being the proportional and resonant gains, $\omega_{1}$ the fundamental frequency and $T_{s}=2 \pi / \omega_{s}$ the controller sampling period, respectively. The resonant filter transfer function corresponds to the impulse-invariant method [15]. $\mathbf{I}$ is a $2 \times 2$ unity matrix, that means that $\mathbf{K}(z)$ is diagonal [16].

The control action calculation also includes an $\mathbf{E}_{c}$ voltage feedforward double path, with the following objectives: 1) provide a filtered value of the main grid component to improve the initial transient [4] and 2) an active damping action based on capacitor voltage derivative term [5], [11]. The active damping action using a backward-Euler discretization is

$$
\mathbf{F}(z)=\left(k_{a d} \frac{1-z^{-1}}{T_{s}}\right) \mathbf{I} .
$$

with $k_{a d}$ being the active damping gain [11].

The system delay is modeled by a time latency $t_{L}$ due to discrete-time operation (e.g., A/D and D/A conversion times of the digital board) and half a control sample due to PWM/ZOH operation; in practice $t_{L}=T_{s}$ is a good approximation (1.5Ts is the total delay) [5], [15]. Furthermore, ZOH and PWM operation also introduce non-linearities, which limit the control action [12], as analyzed in the next section.

The system plant is represented in the S-domain by $\mathbf{P}(s)$. If the grid model is neglected, i.e., $\mathbf{Z}_{g}^{\prime}(s) \approx 0, \mathbf{P}(s)$ can be expressed as a function of LCL parameters [11]. However, this is not an accurate representation of complex power systems, in which, $\mathbf{P}(s)$ is high order and presents resonances [2], [3].

Until now, the matrix notation in the figure represents the three-phase and scalar variables of the real circuit. Subsequently, for the sake of generality scalar notation is used, as 
no couplings between phases are considered. This assumption is accurate for $\alpha \beta$-frame, as both the controller and plant are diagonal [7], [16].

\section{Impedance Stability Criterion and Design for Passivity}

Fig. 1(c) shows an alternative formulation for the VSC current control problem. The converter controller and main filter, which are parametrized during the design stage, are modeled by an equivalent admittance $Y_{c}(\omega)$ [and the closed loop gain $G_{c}(\omega)$ which sets the dependence on the current reference]. The rest of the grid is modeled as $Z_{g}(\omega)$. According to the impedance stability criterion, $Y_{c}(\omega) Z_{g}(\omega)$ Nyquist trajectories set the system dynamics [5], [6].

A key feature of the Impedance/Admittance formulation for design is that $Y_{c}(\omega)$ definition includes all the control actions of the system [17]. Design for passivity is then posed as follows: by assuming that $Z_{g}(\omega)$ is formed by passive components, i.e., is passive, absolute stability of the system defined by $Z_{g}(\omega) Y_{c}(\omega)$ is assured if $Y_{c}(\omega)$ is also passive [4], [5]. In practice, design for passivity aims to define converter control actions that shape $Y_{c}(\omega)$ to be passive in a specified range of frequencies [4], [5].

\section{Region of EfFective Control Action}

\section{A. LCL Filter Operation}

An important observation when considering LCL gridconnected VSC is that the outer admittance (e.g., as seen after the wind turbine transformer), defined as $Y_{g}(\omega)$ in Fig. 1(a), sets the dynamics by its interactions with $Z_{g}^{\prime}(\omega)$. In practice, this scheme well models the case of most wind turbine circuits: the grid-connected converter terminals are at a lowvoltage (LV) network that interfaces the medium-voltage (MV) network through a step-up transformer (also rated at the wind turbine power) [14], [18].

From Fig. 1(a), the outer admittance is given by

$$
\underbrace{Y_{g}(\omega)}_{\text {Outer admittance }}=\frac{1}{j \omega L_{t s}+R_{t s}} / / \underbrace{\left[j \omega C_{c p}+Y_{c}(\omega)\right]}_{\text {Inner admittance }}
$$

with the inner admittance being defined by the parallel of the converter admittance $Y_{c}(\omega)$ and the filter capacitor branch ( $R_{c p} \approx 0$ is assumed). By inspection of (4), it should be noted that only $Y_{c}(\omega)$ models the converter control actions [17], but the remaining $Y_{g}(\omega)$ components are passive. As the capacitive branch admittance increases with frequency, the assumption $\left|j \omega C_{c p}\right|>>\left|Y_{c}(\omega)\right|$ becomes reasonable at frequencies above $\omega_{\text {res }}$; i.e., for high frequency components, the capacitor branch mainly sets the admittance as seen from $E_{c}$ to the VSC. The physical meaning and its implications on stability are clear: the high frequency components in the grid side mainly flow through the capacitor branch and hardly reach the VSC current path; therefore, the control actions derived from the disturbance are minimum and not enough to cause a positive feedback or instability. In order to fulfil this assumption, $\left|Y_{c}(\omega)\right|$ should be small at high frequencies, which is achieved with the proposed method.

From a practical point of view, evaluation of passivity at $Y_{g}(\omega)$ point means to consider the step-up transformer

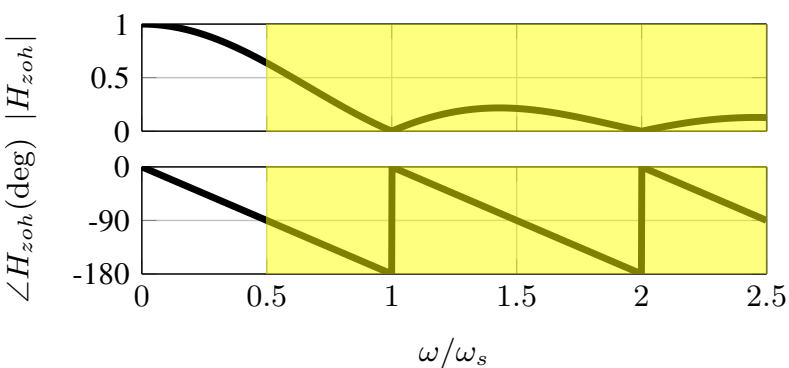

Fig. 2. ZOH frequency response. The yellow zone corresponds to alias region.



(a)

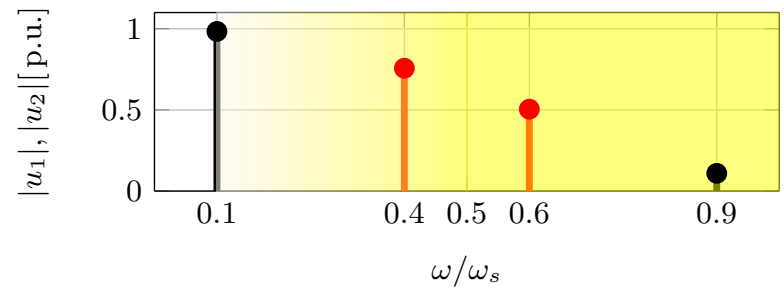

(b)

Fig. 3. Non-linear generation of alias in the ZOH: (black) relativelylow frequency references that generate high frequency and low amplitude alias; (red) references of frequency near to $f_{s} / 2$ generate high amplitude alias in a close frequency which makes difficult its decoupling. (a) timedomain representation; (b) frequency domain representation; the shadow area qualitatively represents how control action effectiveness is gradually lost.

model as a part of the VSC, which is the case of wind turbine applications: the IEC61400 standard includes the stepup transformer as a part of the wind turbine for the low voltage ride through tests (and gives freedom to the wind turbine supplier to select the point for measurement) [18].

\section{B. ZOH, Aliasing and Alias Generation}

In digital controlled system, the control action effectiveness is highly reduced as the frequency is increases [12]. In practice, the control action effectiveness is reduced well below $\omega_{s} / 2$ [8], [19]. ZOH operation and aliasing limit the control action, as explained in the following. The frequency response of the $\mathrm{ZOH}$ block is defined by [8]

$$
H_{z o h}(\omega)=\frac{1-e^{-j \omega T_{s}}}{j \omega T_{s}} .
$$

Fig. 2 shows the ZOH frequency response. In principle, this represents a linear expressions, but when considering the discrete device acquisition, the region highlighted in yellow 
corresponds to alias components [8], [19]. Anti-aliasing filters are then designed so the band of frequencies around $\omega_{s} / 2$ are well attenuated, which implies a high order analog filter with cut-off frequency well below $\omega_{s} / 2$ [19]. The low pass filtering action is at the cost of adding a significant phase-delay in the closed loop, and hence impairs the stability properties. Then, in practice, the frequency region at which anti-aliasing is effective should be out of the control design objective [19].

A highly related non-linear effect of $\mathrm{ZOH}$ arises when analyzing the continuous domain spectrum of a discrete signal generated in the control device. The low frequency reference creates alias at such frequencies, for which the amplitude and (relative) phase angle are also defined by (5) [8], [19]. This effect is represented in Fig. 3 for a relatively low frequency component of $0.1 \omega_{s}$ and for a component near to the Nyquist frequency $0.4 \omega_{s}$. In the first case, it can be assumed that the high frequency alias at $0.9 \omega_{s}$ can be easily filtered by the output filter and anti-aliasing filters spurious free dynamic region. However, this assumption is not reliable in the latter scenario. Therefore, from the physical behavior of the discretetime controller, it is convenient that the control action to be band-limited well below $\omega_{s} / 2[8]^{1}[19]$.

\section{PWM Harmonics}

Besides the ZOH operation, it should be noted that PWM operation is highly nonlinear and generates voltage harmonics at multiple frequencies, which are dependent on different parameters such as: 1) fundamental component amplitude; 2) fundamental component phase angle and 3) carrier phase angle [20]. Fig. 4 shows an example of non-linear and unbalanced generation of harmonics: from a basic modulation strategy working in open loop and using an ideal reference, it can be seen how there are a non-linear generation of harmonic components. The non-linear generation of harmonics due to dead-times can be placed in this category.

Proper control action has been found effective for compensation of non-linear generated low order harmonics: e.g., by resonant controllers [21]. However, the closed loop control ability becomes poor as the frequency increases ${ }^{2}$. At frequencies at which the control action is not effective the phase angle of the measured harmonics depends on non-linear components. In such a circumstance, the impedance stability criterion (i.e., a linear-based control analysis) becomes no longer reliable to asses the system stability, since the measured converter admittance is highly dependent on non-linear harmonics.

\section{Passivity Region Delimitation}

The previous subsections are summarized in the following. The "inner admittance decoupling" provided by the LCL filter isolates high frequency components from the grid. The PWM and $\mathrm{ZOH}$ associated effects also influence the control action

\footnotetext{
${ }^{1}$ The regular sampling technique that avoids components at $\omega_{s} / 2$ can be regarded as a particular case of this effect: two signals of equal amplitude and opposite phase are summed and hence canceled in the acquisition [8].

${ }^{2}$ The dependence between carrier phase-angle is employed to cancel switching harmonics in interleaved converters [14]. This solution can be classified as open loop since the carrier phase-shift angles come from an off-line analysis; i.e., the control action is not calculated in a closed-loop.
}

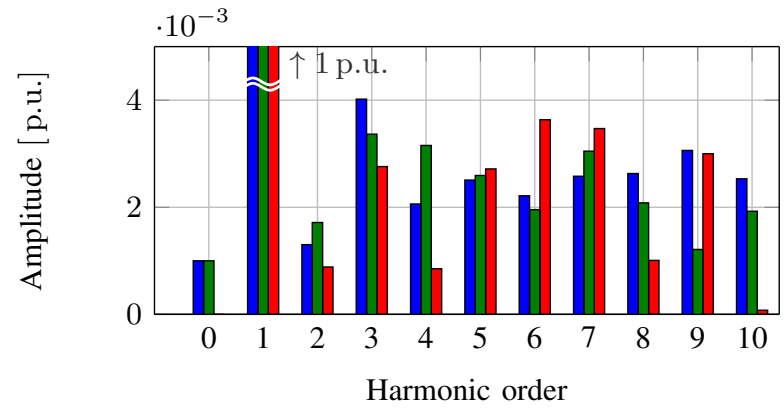

Fig. 4. Non-linear generation of harmonics in three-phase voltages due to PWM operation. Per-phase magnitudes are represented for harmonics. The data has been obtained using a normalized and balanced reference (1 p.u. amplitude at $50 \mathrm{~Hz})$ and a triangular carrier-based PWM $\left(f_{s}=5 \mathrm{kHz}\right.$, $f_{s w}=2.5 \mathrm{kHz}$ ). Unbalance is also present in phase angles (not shown).

effectiveness. From these effects, it is clear that the region at which the converter is effective is well below $\omega_{s} / 2$. As shown in the next sections, $\omega_{s} / 4$ can be set as a reasonable upper limit for design for $Y_{c}(\omega)$ shaping, as $Y_{g}(\omega)$ becomes independent from $Y_{c}(\omega)$ at high frequencies.

\section{Proposed Modeling And Design Criterion}

This section shows the derivation of linear low order expressions for the converter admittance $Y_{c}(\omega)$ and $Y_{g}(\omega)$, that permits a reliable design for passivity. The following assumptions are made in order to simplify the design.

- As discussed in section III, ZOH and PWM effects provide a non-linear generation of alias and harmonics. Even though this non-linearities are not arbitrary as they depend on fundamental component references, they behave as a disturbance in the frequency region for design.

- An the range of frequencies at which a passive behaviour of the current controller is wanted (i.e., above the theoretical closed loop bandwidth), $i_{\text {ref }}=0$ is a reasonable assumption (e.g., no references from outer loops).

- Since $Y_{c}(\omega)$ is considered linear in the design region, its analytical expression is calculated by an $E_{c}$ impulse response (i.e., for mathematical purposes this point can be considered connected to a stiff grid), as follows

$$
Y_{c}(\omega)=\mathcal{L}\left\{\left.i_{c}(t)\right|_{E_{c}(t)=\delta(t), i_{\text {ref }}=0}\right\} .
$$

- The effect of anti-aliasing filters is negligible in the region for design, i.e. up to $\omega_{s} / 4$ in this work.

- The resonant controller (very high gain and selectivity at the fundamental component) has a negligible effect in the range of frequencies for a passive design (frequencies beyond the theoretical closed loop bandwidth) [15].

Fig. 5(a) depicts the hybrid system that represents $Y_{c}(\omega)$, which is derived from Fig. 1(b) by applying these assumptions. The Z-domain expression is obtained by considering the sample and hold effects in the discretization of the plant: the control paths that drive into the discrete device are discretized by $\mathrm{ZOH}$ method, meanwhile the Tustin method is accurate to represent the dynamics only due to the disturbance [7], [8]. Fig. 5(b) represents the derived converter admittance in the 


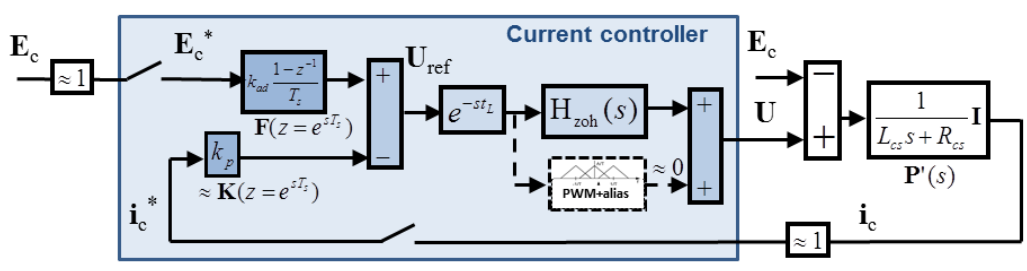

(a)

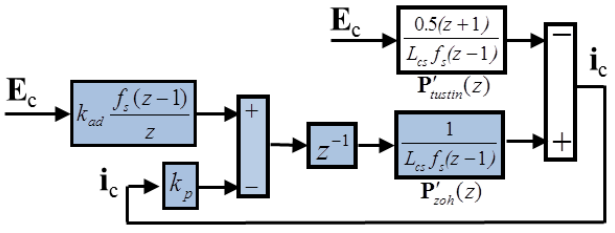

(b)

Fig. 5. Converter admittance modeling. (a) Admittance model from hybrid system discarding non-linear PWM harmonics and aliasing effects, and ideal perturbation at $E_{c}$ point. (b) Proposed reduced order in the discrete domain for $Y_{c}(z)$ shaping.

TABLE I

Physical System Parameters

\begin{tabular}{|c|c|}
\hline Parameter & Value \\
\hline Rated Power & $S=700 \mathrm{kVA}$ \\
\hline Rated Voltage (Line to line RMS) & $V=690 \mathrm{~V}$ \\
\hline Switching/Sampling frequency & $f_{s w}=2.5 \mathrm{kHz}, f_{s}=5 \mathrm{kHz}$ \\
\hline Converter (primary) inductance & $L_{c s}=0.1 \mathrm{p} . \mathrm{u}$. \\
\hline Converter equivalent resistance & $R_{c s}=0.01 \mathrm{p} . \mathrm{u}$. \\
\hline Capacitor & $C_{c p}=0.06 \mathrm{p} . \mathrm{u}$. \\
\hline Passive damping ESR & $R_{c p}=0.001$ p.u. \\
\hline Grid Side Inductance (Trafo leakage) & $L_{t s}=0.1 \mathrm{p.u}$. \\
\hline Grid Side Resistor & $R_{t s}=0.01 \mathrm{p} . \mathrm{u}$. \\
\hline
\end{tabular}

Z-domain, $Y_{c}(z)$. Its analytical derivation is

$$
Y_{c}(z)=\frac{i_{c}(z)}{E_{c}(z)}=\frac{-0.5 z^{3}-0.5 z^{2}+k_{a d} f_{s} z-k_{a d} f_{s}}{z\left[L_{c s} f_{s}\left(z^{2}-z\right)+k_{p}\right]} .
$$

This expression is third order and biproper, with zeros and poles depending on $k_{a d}$ and $k_{p}$, respectively. The outer admittance $Y_{g}(\omega)$ can be calculated by (4) with $Y_{c}(\omega)=Y_{c}\left(z=e^{j \omega T_{s}}\right)$.

A convenient combination of $k_{a d}$ and $k_{p}$ provides a stable pole/zero cancellation, which reduces the $Y_{c}(z)$ order to a first one. This procedure inherently enhances the passivity as it reduces the phase variations in the $Y_{c}(z)$ frequency response [i.e., phase variations associated to poles and zeros at the region of interest]. In practice, this simple design criterion assures $Y_{c}(z)$ passivity for frequencies below $\omega_{s} / 4$ (and beyond), as proved in the next section.

\section{Case Study and Simulation Results}

The proposed design approach has been applied using the data of Table I. Fig. 6 shows the pole and zero trajectories of $Y_{c}(z)$, which depend on $k_{a d}$ and $k_{p}$, respectively. By a parametric analysis, it has been found that the set $\left(k_{p}=0.58, k_{a d}=1.33 e^{-4}\right)$ provides two pole-zero cancellations (complex pairs). With this tuning, $Y_{c}(z)$ is reduced to a first order expression. $Y_{c}(z)$ has a pole at $z=0$, which represents one-sample delay effect: a -90 deg delay at $\omega_{s} / 4$ is introduced. $Y_{c}(z)$ has also a nonminimum phase zero at $z \approx-2$, which provides some phase-lead ${ }^{3}$ and extends the passivity region slightly beyond $\omega=\omega_{s} / 4$.

Fig. 7 and 8 show the frequency responses according to (7) and (4), respectively (together with simulation results points, which are also explained below). As aimed, 1) a passive

\footnotetext{
${ }^{3} \mathrm{~A}$ nonminimum phase zeros does not totally compensate a pole delay [8].
}

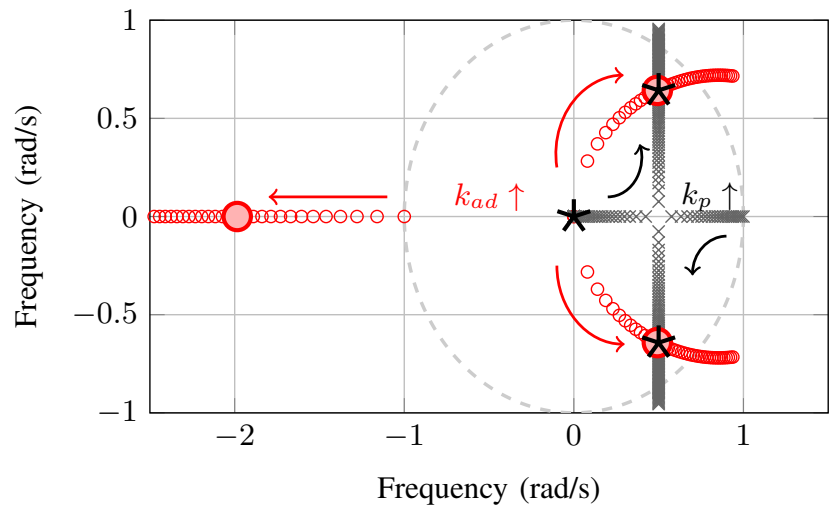

Fig. 6. Design for passivity based on $Y_{c}(z)$ order reduction. The highlighted roots show the tuning which provides the aimed pole-zero cancellation.

resistivity of $Y_{c}(\omega)$ [i.e., $90 \mathrm{deg}>\angle Y_{c}(\omega)>-90 \mathrm{deg}$ ] is obtained from low frequencies to beyond $\omega_{s} / 4$;2) $Y_{g}(\omega)$ becomes inductive at high frequencies [i.e., $\angle Y_{g}(\omega) \approx-90 \mathrm{deg}$ from around $0.2 \omega_{s}$ ]: i.e., the LCL capacitance branch is much more dominant than $Y_{c}(\omega)$ at high frequencies.

Simulation results have been obtained with Simulink (discrete controller working at $T_{s}$ ) and PLECS blockset (2 level VSC, switching model). In order to get the admittances by simulation, the procedure of EN-50388 is performed as follows [13]: 1) for each point, a voltage harmonic component is programmed at $E(t) ; 2)$ Time domain simulation is performed, until reaching a steady state ${ }^{4}$; 3 ) The harmonic spectrum of grid and converter currents is obtained (magnitude and phase) and 4) The admittances are measured by the formulae

$$
Y_{c, g}(\omega)=\frac{\left|i_{c, g}(\omega)\right|^{\mathrm{FFT}}}{\left|E_{c, g}(\omega)\right|^{\mathrm{FFT}}}\left[\angle \phi_{i_{c, g} \mathrm{FT}}^{\mathrm{FF}}-\angle \phi_{E_{c, g}(\omega)}^{\mathrm{FF}}\right]
$$

with the FFT superscript meaning data obtained by spectrum analysis. It can be appreciated that an overall good matching is obtained for $Y_{c}(\omega)$, even though some deviations around $0.12 \omega_{s}(\approx$ LCL resonance frequency) are found; the main reason for such deviations is the fact that non-linear PWM harmonics are amplified by the LCL resonance. For $Y_{g}(\omega)$, the matching is good in all the range of frequencies, which proves the main hypothesis: at high frequencies, the LCL capacitor branch well decouples the grid-side perturbation (i.e., grid voltage harmonics) from the VSC current path and hence,

\footnotetext{
${ }^{4}$ Due to space constraints, these curves are not included in the paper.
} 

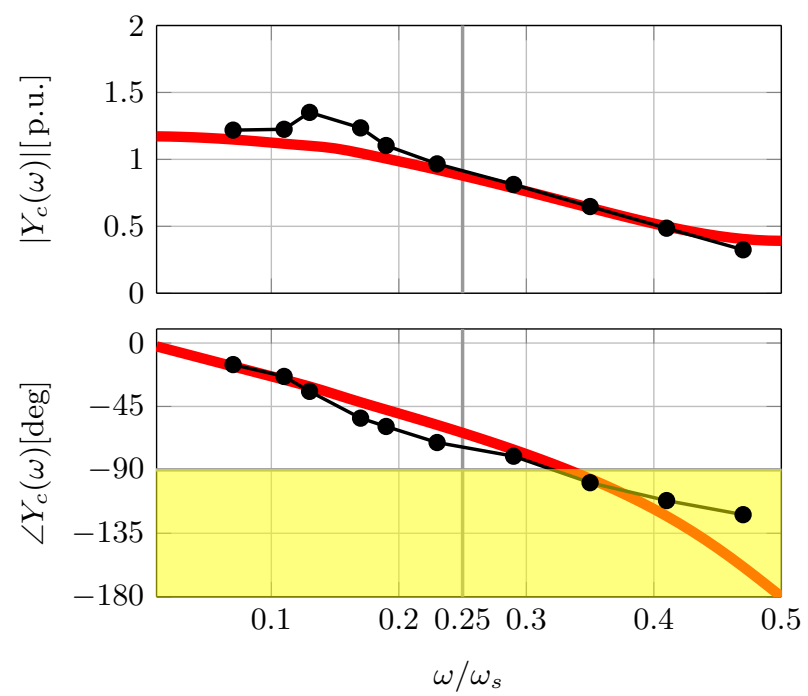

Fig. 7. $Y_{c}(\omega)$ theoretical (red) and obtained by simulation (black). For the simulation $Z_{g}^{\prime}(\omega), L_{t s}(\omega) \approx 0 \Rightarrow E_{c}(t) \approx E(t)$ [see (6)].

avoids any potential positive feedback action caused by it.

\section{CONClusions}

Design for passivity is approached as a reliable method to assure the stability of LCL grid-connected VSCs working in power systems with potential resonances. In comparison with existing works, the frequency region for design is reconsidered by having into account non-linear ZOH/PWM effects and the nature of the LCL filter topology. It has been found that the LCL filter is convenient for stability, as the capacitor well isolates high frequency disturbance from the VSC current path. This remark also eases the controller design as the frequency region for analysis can be safely reduced without compromising the stability goal. Subsequently, the VSC is modeled by a Z-domain admittance and a systematic design procedure is formulated as shaping it to be first order, which implies the VSC is passive in the (relatively) low frequency region. The feasibility and accuracy of the proposed methodology is proved by simulation results (PLECS switching model).

\section{REFERENCES}

[1] D. Dujic, C. Zhao, A. Mester, J. K. Steinke, M. Weiss, S. LewdeniSchmid, T. Chaudhuri, and P. Stefanutti, "Power electronic traction transformer-low voltage prototype," IEEE Trans. Power Electron., vol. 28, no. 12, pp. 5522-5534, 2013.

[2] M. Bradt, B. Badrzadeh, E. Camm, D. Mueller, J. Schoene, T. Siebert, T. Smith, M. Starke, and R. Walling, "Harmonics and resonance issues in wind power plants," in Proc. of the IEEE PES GM, 2011.

[3] H. Kocewiak, J. Hjerrild, and C. Bak, "Wind turbine converter control interaction with complex wind farm systems," IET Renewable Power Generation, vol. 7, no. 4, pp. 380-389, 2013.

[4] L. Harnefors, L. Zhang, and M. Bongiorno, "Frequency-domain passivity-based current controller design," IET Power Electron., vol. 1, no. 4 , pp. $455-465$, Dec. 2008

[5] L. Harnefors, A. G. Yepes, A. Vidal, and J. Doval-Gandoy, "Passivitybased controller design of grid-connected vscs for prevention of electrical resonance instability," IEEE Trans. Ind. Electron., vol. 62, no. 2, pp. 702-710, 2015.

[6] J. Sun, "Impedance-based stability criterion for grid-connected inverters," IEEE Trans. Power Electron., vol. 26, no. 11, pp. 3075-3078, 2011.
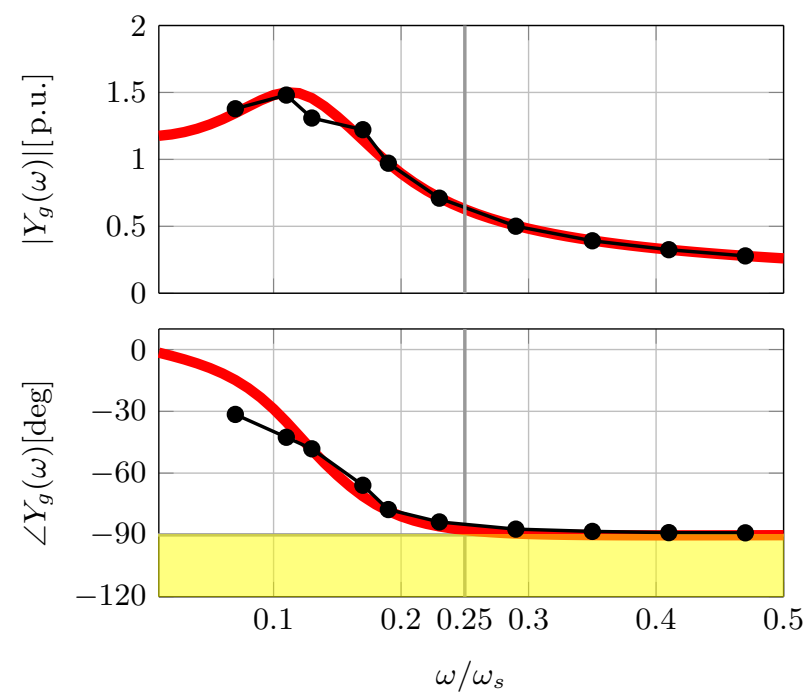

Fig. 8. $Y_{g}(\omega)$ theoretical (red) and obtained by simulation (black). For the simulation $Z_{g}^{\prime}(\omega) \approx 0 \Rightarrow E_{g}(t) \approx E(t)$.

[7] G. C. Goodwin, S. F. Graebe, and M. E. Salgado, Control System Design. Prentice Hall, 2000.

[8] G. F. Franklin, J. D. Powell, and M. L. Workman., Digital Control of Dynamic Systems (3rd Edition), ., Ed. Addison-Wesley, 1997.

[9] L. Corradini, D. Maksimović, P. Mattavelli, and R. Zane, Digital Control of High-frequency Switched-mode Power Converters. John Wiley \& Sons, 2015.

[10] Z. Xin, X. Wang, P. C. Loh, and F. Blaabjerg, "Realization of digital differentiator using generalized integrator for power converters," IEEE Trans. Power Electron., vol. 30, no. 12, pp. 6520-6523, 2015.

[11] J. Dannehl, F. Fuchs, S. Hansen, and P. Thogersen, "Investigation of active damping approaches for pi-based current control of gridconnected pulse width modulation converters with LCL filters," IEEE Trans. Ind. Appl., vol. 46, no. 4, pp. 1509-1517, 2010.

[12] C. M. Wolf, M. W. Degner, and F. Briz, "Analysis of current sampling errors in pwm vsi drives," IEEE Trans. Ind. Appl., vol. 51, no. 2, pp. $1551-1560,2015$

[13] EN50388. Railway Applications - Power supply and rolling stock Technical criteria for the coordination between power supply (substation) and rolling stock to achieve interoperability, Cenelec Std., 2012.

[14] G. Gohil, L. Bede, R. Teodorescu, T. Kerekes, and F. Blaabjerg, "Line filter design of parallel interleaved vscs for high-power wind energy conversion systems," IEEE Trans. Power Electron., vol. 30, no. 12, pp. 6775-6790, 2015.

[15] A. Vidal, F. D. Freijedo, A. G. Yepes, J. Malvar, O. Lopez, and J. Doval-Gandoy, "Transient response evaluation of stationary-frame resonant current controllers for grid-connected applications," IET Power Electronics, vol. 7, no. 7, pp. 1714-1724, 2014.

[16] F. D. Freijedo, A. Vidal, A. G. Yepes, J. M. Guerrero, O. Lopez, J. Malvar, and J. Doval-Gandoy, "Tuning of synchronous-frame pi current controllers in grid-connected converters operating at a low sampling rate by MIMO root locus," IEEE Trans. Ind. Electron., vol. 62, no. 8, pp. 5006-5017, 2015.

[17] L. Harnefors, X. Wang, A. G. Yepes, and F. Blaabjerg, "Passivity-based stability assessment of grid-connected vscs-an [::overview::]," IEEE Journal of Emerging and Selected Topics in Power Electronics, 2016.

[18] IEC 61400-21 Ed.2, Wind turbines - Part 21: Measurement and assessment of power quality characteristics of grid connected wind turbines, IEC Std., Aug. 2008.

[19] K. Sozanski, Digital Signal Processing in Power Electronics Control Circuits. Springer Verlag, 2013.

[20] D. G. Holmes and T. A. Lipo, Pulse Width Modulation for Power Converters, M. El-Hawary, Ed. IEEE Press Series, 2003.

[21] A. Kulkarni and V. John, "Mitigation of lower order harmonics in a grid-connected single-phase pv inverter," IEEE Trans. Power Electron., vol. 28, no. 11, pp. 5024-5037, Nov 2013. 